Pacific

Journal of

Mathematics

BESSEL DISTRIBUTIONS FOR GL(3) OVER THE $p$-ADICS

Ehud Moshe Baruch

Volume $217 \quad$ No. 1

November 2004 


\title{
BESSEL DISTRIBUTIONS FOR GL(3) OVER THE $p$-ADICS
}

\author{
Ehud Moshe Baruch
}

\begin{abstract}
We show that the Bessel distribution attached to a generic representation of $\operatorname{GL}(3, F)$, where $F$ is a $p$-adic field, is given by a Bessel function.
\end{abstract}

\section{Introduction and main results}

This is the second part of our study of Bessel functions and Bessel distributions on $\operatorname{GL}(3, F)$, where $F$ is a nonarchimedean local field. In the first part, $[\mathbf{1}]$, we attached Bessel functions to every generic representation of $\mathrm{GL}(3, F)$ and showed that their asymptotics are given by certain orbital integrals. Here we show that these orbital integrals give rise to locally integrable functions. Hence the Bessel functions are locally integrable. We also prove a kernel formula in the Whittaker model involving the Bessel function. This kernel formula will allow us to show that the Bessel distributions are given by Bessel functions, which is our main result.

1.1. Main results. We state here our main results. We will use the notations introduced in $[\mathbf{1}]$, which we recall now.

Let $G=\mathrm{GL}(3, F)$ and let $B$ be the Borel subgroup of upper triangular matrices, $A$ the subgroup of diagonal matrices, and $Z$ the center of $G$. Let $N$ be the subgroup of upper unipotent matrices and $\bar{N}$ the subgroup of lower unipotent matrices. Let $\mathbb{W}=N(A) / A$ be the Weyl group, where $N(A)$ is the normalizer of $A$. We identify $\mathbb{W}$ with the set of six permutation matrices in $N(A)$. Let

$$
w_{0}=\left(\begin{array}{lll} 
& & 1 \\
& 1 & \\
1 & &
\end{array}\right)
$$

be the longest Weyl element in $\mathbb{W}$. Let $\psi_{F}$ be a nontrivial character of $F$ and let $\psi$ be a character of $N$ defined by

$$
\psi(n)=\psi_{F}\left(n_{1,2}+n_{2,3}\right),
$$

where $n_{i, j}$ is the $i, j$ entry of $n \in N$. It is clear that

$$
\psi\left(w_{0}^{t} n w_{0}\right)=\psi(n)
$$


for all $n \in N$. Let $\omega$ be a quasicharacter of $Z$. Let $f \in C_{c}^{\infty}(G)$ and $g \in \bar{N} B$. We define

$$
I_{f, \omega, \psi}(g)=\int f\left({ }^{t} n_{1} z g n_{2}\right) \omega^{-1}(z) \psi^{-1}\left(n_{1}\right) \psi^{-1}\left(n_{2}\right) d n_{1} d z d n_{2} .
$$

It follows from [7] that this integral is absolutely convergent for every $g$ in $\bar{N} B$. We extend $I_{f, \omega, \psi}$ to a function on $G$ by setting $I_{f, \omega, \psi}(g)=0$ when $g \notin \bar{N} B$.

Theorem 1.1. $I_{f, \omega, \psi}$ is locally integrable as a function on $G$.

Define

$$
J_{f, \omega, \psi}(g)=\int f\left(n_{1} z g n_{2}\right) \omega^{-1}(z) \psi^{-1}\left(n_{1}\right) \psi^{-1}\left(n_{2}\right) d n_{1} d z d n_{2} .
$$

This integral converges absolutely for every $g \in B w_{0} B$. We extend it to a function on $G$ as above.

Corollary 1.2. $J_{f, \omega, \psi}$ is locally integrable as a function on $G$.

Proof. Let $f_{w_{0}}(g)=f\left(w_{0} g\right)$. If $f \in C_{c}^{\infty}(G)$ then $f_{w_{0}} \in C_{c}^{\infty}(G)$, hence by Theorem 1.1, $I_{f_{w_{0}}, \omega, \psi}$ is a locally integrable function. It follows that the function

$$
g \mapsto I_{f_{w_{0}}, \omega, \psi}\left(w_{0} g\right)
$$

is locally integrable. Assume that $g \in B w_{0} B$. Using (1.2) we have

$$
\begin{aligned}
I_{f_{w_{0}, \omega, \psi}\left(w_{0} g\right)} & =\int f\left(w_{0}^{t} n_{1} w_{0} g z n_{2}\right) \omega^{-1}(z) \psi^{-1}\left(n_{1}\right) \psi^{-1}\left(n_{2}\right) d n_{1} d z d \bar{n}_{2} \\
& =\int f\left(n_{1} z g n_{2}\right) \omega^{-1}(z) \psi^{-1}\left(n_{1}\right) \psi^{-1}\left(n_{2}\right) d n_{1} d z d n_{2} \\
& =J_{f, \omega, \psi}(g) .
\end{aligned}
$$

Let $(\pi, V)$ be a generic irreducible admissible representation of $G$, and $\omega_{\pi}$ the central character of $\pi$. Let $j_{\pi, \psi}$ be the Bessel function defined in $[\mathbf{1}, 6.5]$.

Corollary 1.3. $j_{\pi, \psi}$ is a locally integrable function on $G$.

Proof. By [1, Theorem 1.7], for every $g \in G$ there exist an open compact neighborhood $U$ of $g$ and a function $f \in C_{c}^{\infty}(G)$ such that $j_{\pi, \psi}=J_{f, \omega_{\pi}, \psi}$ as functions on $U$. Since $J_{f, \omega_{\pi}, \psi}$ is locally integrable for every $f \in C_{c}^{\infty}(G)$ the result follows.

Remark 1.4. It is clear from the relation between $J_{f, \omega, \psi}$ and $I_{f, \omega, \psi}$ that it is enough to study one of these integrals. We will focus on $I_{f, \omega, \psi}$ in order to match the notation of [7] and [8], which studies the asymptotics of $I_{f, \omega, \psi}$.

Let $\mathcal{W}(\pi, \psi)$ be the Whittaker model of $\pi$ (see (5.1)) and let $\mathcal{W}^{0}(\pi, \psi)$ be the subspace defined in [1, Definition 4.1]. Let $N_{2}$ be the subgroup of upper unipotent matrices in $\mathrm{GL}(2, F), B_{2}$ the subgroup of upper triangular matrices in $\operatorname{GL}(2, F)$ and $w$ a $\operatorname{GL}(2)$ Weyl element (see Section 5). 
Theorem 1.5. Let $W \in \mathcal{W}^{0}(\pi, \psi)$. Let $g \in B_{2} w B_{2}$. Then

$$
W\left(\begin{array}{ll}
g & 1
\end{array}\right)=\int_{N_{2} \backslash \mathrm{GL}(2, F)} j_{\pi, \psi}\left(\begin{array}{ll}
g h^{-1} & 1
\end{array}\right) W\left(\begin{array}{ll}
h & \\
& 1
\end{array}\right) d h .
$$

Remark 1.6. If $\pi$ is supercuspidal then $\mathcal{W}^{0}(\pi, \psi)=\mathcal{W}(\pi, \psi)$. By changing $W$ in (1.4) to

$$
\pi\left(\begin{array}{ll}
h & \\
& 1
\end{array}\right) W
$$

it is easy to show that (1.4) holds for every $W \in \mathcal{W}(\pi, \psi)$ and every $g \in$ $\mathrm{GL}(2, F)$. This gives a kernel formula for the action of the Weyl elements in the Kirillov model of a supercuspidal representation.

Finally, the main theorem of our paper is the following. Let $J_{\pi, \psi}$ be the Bessel distribution defined in [2] (see (6.3)).

Theorem 1.7. $J_{\pi, \psi}(f)=\int_{G} j_{\pi, \psi}(g) f(g) d g$ for all $f \in C_{c}^{\infty}(G)$.

\section{Notations and preliminaries}

We recall some notations from [1]. Let $F$ be a nonarchimedean local field. Let $O$ be the ring of integers in $F$ and let $P$ be the maximal ideal in $O$. Let $\varpi$ be a generator of $P$. We denote by $|x|$ the normalized absolute value of $x \in F$. Let $q=|O / P|$ be the order of the residue field of $F$. Then $|\varpi|=q^{-1}$. Let $G=\mathrm{GL}(3, F)$ and $K=\mathrm{GL}(3, O)$. Let $A$ be the subgroup of diagonal matrices in $G$ consisting of matrices of the form

$$
d\left(a_{1}, a_{2}, a_{3}\right)=\left(\begin{array}{ccc}
a_{1} & & \\
& a_{2} & \\
& & a_{3}
\end{array}\right), \quad a_{1}, a_{2}, a_{3} \in F^{*} .
$$

We let

$$
Z=Z(G)=\left\{d(x, x, x): x \in F^{*}\right\} .
$$

Let $X=X(A)=\operatorname{Hom}_{F}(A, F)$ be the group of rational homomorphisms. Then each $\alpha \in X(A)$ is of the form

$$
\alpha_{n_{1}, n_{2}, n_{3}}\left(d\left(a_{1}, a_{2}, a_{3}\right)\right)=a_{1}^{n_{1}} a_{2}^{n_{2}} a_{3}^{n_{3}},
$$

with $n_{1}, n_{2}, n_{3} \in \mathbf{Z}$. Let $X^{\vee}=X^{\vee}(A)=\operatorname{Hom}_{F}(F, A)$. Then each $\alpha^{\vee} \in X^{\vee}$ is of the form

$$
\alpha_{n_{1}, n_{2}, n_{3}}^{\vee}(x)=d\left(x^{n_{1}}, x^{n_{2}}, x^{n_{3}}\right), \quad x \in F .
$$

For each $\alpha \in X$ we let $|\alpha|: A \rightarrow \mathbf{R}$ and $\alpha^{\vee} \in X^{\vee}$ be the obvious maps.

For $i, j \in\{1,2,3\}, i \neq j$, we let $\alpha_{i, j}: A \rightarrow F$ be the functions defined by

$$
\alpha_{i, j}\left(d\left(a_{1}, a_{2}, a_{3}\right)\right)=\frac{a_{i}}{a_{j}} .
$$


Let $\Phi=\left\{\alpha_{i, j}\right\}$ be the root system of $G$ and let $\Phi^{\vee}=\left\{\alpha^{\vee}: \alpha \in \Phi\right\}$. We have $\Phi=\Phi^{+} \cup \Phi^{-}$, where $\Phi^{+}=\left\{\alpha_{i, j}: i<j\right\}$ is the set of positive roots and $\Phi^{-}$is the set of negative roots. Similarly, $\Phi^{\vee}=\Phi^{\vee,+} \cup \Phi^{\vee,-}$. Let $\mathcal{B}=\left\{\alpha_{1,2}, \alpha_{2,3}\right\}$ be the set of simple roots.

Finally, if $g \in G$ we let $\Delta_{i}(g)$ be the principal $i \times i$ minor of $g$. We define $\Delta: G \rightarrow \mathbf{R}$ by

$$
\Delta(g)=\left|\frac{\left(\Delta_{1}(g)\right)^{2}\left(\Delta_{2}(g)\right)^{2}}{\left(\Delta_{3}(g)\right)^{2}}\right| .
$$

It is easy to see that $\Delta\left({ }^{t} n_{1} g n_{2}\right)=\Delta(g)$ for every $n_{1}, n_{2} \in N$ and $g \in G$. Also, $\Delta(g)=0$ if $g \notin \bar{N} B$ and

$$
\Delta\left(d\left(a_{1}, a_{2}, a_{3}\right)\right)=\left|a_{1}^{2} a_{3}^{-2}\right| .
$$

\section{3. $\Delta^{-1 / 2+\epsilon}$ is locally integrable}

In this section we shall use the results of Dạbrowski and Reeder, [4], to show that $\Delta^{-1 / 2+\epsilon}$ is locally integrable for every $\epsilon>0$.

Let $f \in C_{c}^{\infty}(G)$ and let $g \in \bar{N} B$. We define the orbital integral:

$$
O_{f}(g)=\int_{N \times N} f\left({ }^{t} n_{1} g n_{2}\right) d n_{1} d n_{2} .
$$

The convergence of this integral follows from [7]. Dạbrowski and Reeder studied this integral when $f=f_{0}$ is the characteristic function of the maximal compact subgroup $K$. For each $a \in A$ there exist a unique $\lambda_{a} \in X^{\vee}$ such that $a=a_{K} \lambda_{a}(\varpi)$, where $a_{K} \in A_{K}=A \cap K$.

Theorem $3.1([4,0.4])$. Let $f_{0}$ be the characteristic function of $K$. Then $O_{f_{0}}(a)=0$ if $\lambda=\lambda_{a} \notin \mathbb{Z}_{\geq 0} \Phi^{\vee,+}$, i.e., $\lambda_{a}$ is not a nonnegative integral linear combination of positive coroots. If $\lambda_{a}$ is such a linear combination we write

$$
\lambda_{a}=\sum_{\beta \in \Phi^{\vee},+} m_{\beta} \beta=m_{1,2} \alpha_{1,2}^{\vee}+m_{2,3} \alpha_{2,3}^{\vee}+m_{1,3} \alpha_{1,3}^{\vee}, \quad m_{i, j} \geq 0,
$$

and $\underline{m}=\left(m_{1,2}, m_{2,3}, m_{1,3}\right)=\left(m_{\beta}\right)_{\beta \in \Phi^{\vee},+}$. Then

$$
O_{f_{0}}(a)=\Delta^{-1 / 2}(a) \sum_{\underline{m}}(1-1 / q)^{\kappa(\underline{m})},
$$

where $\kappa(m)$ is the number of strictly positive coordinates of $\underline{m}$, and $\underline{m}$ runs over all possible decompositions (3.1).

Each $\lambda_{a} \in X^{\vee}$ that can be written as in (3.1) can be written in the form

$$
\lambda_{a}=m_{1}(a) \alpha_{1,2}^{\vee}+m_{2}(a) \alpha_{2,3}^{\vee},
$$

where $m_{1}(a), m_{2}(a)$ are nonnegative integers uniquely determined by $a$. It is easy to see that for such $a$ we have

$$
\Delta(a)=q^{-2\left(m_{1}(a)+m_{2}(a)\right)} .
$$


Corollary 3.2. Assume that $a$ is such that $\lambda_{a}$ can be written as in (3.1). Then

$$
\begin{aligned}
O_{f_{0}}(a) & \leq q^{m_{1}(a)+m_{2}(a)} \max \left(1, m_{1}(a)+m_{2}(a)\right) \\
& =\Delta^{-1 / 2}(a) \max \left(1, \log _{q}\left(\Delta^{-1 / 2}(a)\right) .\right.
\end{aligned}
$$

Proof. Let $R(a)=R\left(\lambda_{a}\right)$ be the number of possibilities of writing $\lambda_{a}$ as in (3.1). Then it follows from (3.2) that

$$
O_{f_{0}}(a) \leq \Delta^{-1 / 2}(a) R(a) .
$$

It is easy to see that $R(a)=\min \left(m_{1}(a), m_{2}(a)\right)+1$. In particular, if $\left(m_{1}(a), m_{2}(a)\right) \neq(0,0)$ then $R(a) \leq m_{1}(a)+m_{2}(a)$. It is clear that if $\left(m_{1}(a), m_{2}(a)\right)=(0,0)$ then $R(a)=1$ and our inequality holds.

The following corollary is essential to the question of local integrability of the Bessel function. This result is analogous to Harish-Chandra's result in $[6]$ that $D^{-1 / 2}$ is locally integrable. (See [6] for the definition of $D$.)

Corollary 3.3. $\Delta^{-1 / 2+\epsilon}$ is locally integrable for every $\epsilon>0$.

Proof. It is enough to show that

$$
\int_{G} \Delta^{-1 / 2+\epsilon}(g) f(g) d g<\infty
$$

for every characteristic function $f$ of $K g_{0}$, where $g_{0} \in G$. Write $g_{0}=k_{0} b_{0}$ for some $b_{0} \in B$ and $k_{0} \in K$. Hence it is enough to show that (3.5) holds for

$$
f=\rho_{r}\left(b_{0}^{-1}\right) f_{0},
$$

where $\rho_{r}$ is right translation. Writing $b_{0}=a_{0} n_{0}$ for $a_{0} \in A$ and $n_{0} \in N$ we have

$$
\begin{aligned}
\int_{G} \Delta^{-1 / 2+\epsilon}(g)\left(\rho_{r}\left(b_{0}\right) f_{0}\right)(g) d g & =\int_{G} \Delta^{-1 / 2+\epsilon}(g) f_{0}\left(g b_{0}^{-1}\right) d g \\
& =\int_{G} \Delta^{-1 / 2+\epsilon}\left(g b_{0}\right) f_{0}(g) d g \\
& =\Delta^{-1 / 2+\epsilon}\left(a_{0}\right) \int_{G} \Delta^{-1 / 2+\epsilon}(g) f_{0}(g) d g .
\end{aligned}
$$

Hence it is enough to prove (3.5) for $f=f_{0}$. Using the invariance properties of $\Delta$ and writing $d g=\Delta(a) d n_{1} d a d n_{2}$ on the set of elements of the form 
${ }^{t} n_{1} a n_{2}$, where $n_{1}, n_{2} \in N$ and $a \in A$, we get

$$
\begin{aligned}
\int_{G} \Delta^{-1 / 2+\epsilon}(g) f_{0}(g) d g & =\int_{N \times A \times N} \Delta^{-1 / 2+\epsilon}\left({ }^{t} n_{1} a n_{2}\right) f_{0}\left({ }^{t} n_{1} a n_{2}\right) \Delta(a) d n_{1} d a d n_{2} \\
& =\int_{A} \Delta^{1 / 2+\epsilon}(a) O_{f_{0}}(a) d a \\
& =\sum_{\lambda \in X^{\vee}} \int_{(A \cap K) \lambda(\varpi)} \Delta^{1 / 2+\epsilon}(a) O_{f_{0}}(a) d a \\
& =\left(\int(A \cap K) d a\right)\left(\sum_{\lambda \in X^{\vee}} \Delta^{-1 / 2+\epsilon}(\lambda(\varpi)) O_{f_{0}}(\lambda(\varpi))\right) .
\end{aligned}
$$

We can assume that $d a$ assigns measure 1 to $A \cap K$. By Theorem 3.1 the sum over $\lambda \in X^{\vee}$ takes place for $\lambda$ of the form

$$
\lambda=m_{1} \alpha_{1,2}^{\vee}+m_{2} \alpha_{2,3}^{\vee},
$$

where $m_{1}$ and $m_{2}$ are nonnegative integers. By Corollary 3.2 we have, for $\lambda$ written as above,

$$
\Delta^{1 / 2+\epsilon}(\lambda(\varpi)) O_{f_{0}}(\lambda(\varpi)) \leq q^{-\epsilon\left(m_{1}+m_{2}\right)} \max \left(1, m_{1}+m_{2}\right) .
$$

Hence our integral is majorized by

$$
1+\sum_{m_{1} \geq 0} \sum_{m_{2} \geq 0} q^{-\epsilon\left(m_{1}+m_{2}\right)}\left(m_{1}+m_{2}\right),
$$

which is finite when $\epsilon>0$.

\section{4. $\psi$-orbital integrals are locally integrable}

In this section we shall use the results of Jacquet and Ye, [7] and [8], to show that the $\psi$ orbital integrals are locally integrable. Consequently, by Corollary 1.3 Bessel functions attached to irreducible representations are locally integrable. Let $I_{f, \psi}$ be the $\psi$ orbital integral defined in [7] by

$$
I_{f, \psi}(g)=\int f\left({ }^{t} n_{1} g n_{2}\right) \psi^{-1}\left(n_{1}\right) \psi^{-1}\left(n_{2}\right) d n_{1} d n_{2} .
$$

Here $g \in \bar{N} B$. Notice that $I_{f, \psi}$ does not have the integration over the center that appears in the definition of $I_{f, \omega, \psi}$ in (1.3).

Let

$$
\begin{gathered}
a_{1}(z)=\left(\begin{array}{ccc}
z & & \\
& -1 / z & \\
& & 1
\end{array}\right), \quad a_{2}(z)=\left(\begin{array}{ccc}
1 & & \\
& z & \\
& & -1 / z
\end{array}\right), \\
a_{1}(x, y)=\left(\begin{array}{lll}
x & & \\
& x & \\
& & y
\end{array}\right), \quad a_{2}(x, y)=\left(\begin{array}{lll}
x & & \\
& y & \\
& & y
\end{array}\right),
\end{gathered}
$$


and

$$
a(x, y)=\left(\begin{array}{lll}
x & & \\
& y & \\
& & -1 / x y
\end{array}\right) .
$$

For $z \neq 0$ we define

$$
K_{1}(z, \psi)=\psi(2 / z) \gamma(2 / z, \psi)|2 z|^{-1 / 2},
$$

where $\gamma(2 / z, \psi)$ is the Weil constant as in [7, Proposition 2.3]. Let $m$ be large enough so that the map $z \mapsto z^{2}$ is an analytic bijection of $1+P^{m}$ to $1+2 P^{m}$. If $\mu \in 1+2 P^{m}$ we denote by $\sqrt{\mu}$ the inverse image of $\mu$ under this map. Let $a, b \in F^{*}$ be such that $|a|<q^{-m}$ and $|b| \leq 1$. Let $\mu=a+b x^{2}$. Define

$$
K_{0}(a, b, \psi)=|a|^{-1}|b|^{-1 / 2} \gamma(1, \psi) \gamma(-b, \psi)(2, b) \int \psi\left(2 x-\frac{2 x}{b \sqrt{\mu}}\right)(x, b) d x,
$$

where the range of integration is $\mu \equiv 1 \bmod 2 P^{m}$.

We define $K_{0}$ on the set $\left\{(a, b): 0 \neq|a|<q^{-m},|a b|<q^{-m},|b|>1\right\}$ by

$$
K_{0}(a, b, \psi)=K_{0}\left(a b, 1 / b, \psi^{-1}\right) .
$$

Now $K_{0}$ is defined for all $a, b \in F^{*}$ such that $|a|<q^{-m}$ and $|a b|<q^{-m}$.

Lemma 4.1. There exist positive constants $B_{1}, B_{2}, B_{3}$ such that:

(1) $\left|K_{1}(z, \psi)\right| \leq B_{1}|z|^{-1 / 2}$.

(2) $\left|K_{0}(a, b)\right| \leq B_{2}|a|^{-3 / 2}|b|^{-1 / 2}, \quad$ if $|a|<q^{-m},|a b|<q^{-m},|b|=1$.

(3) $\left|K_{0}(a, b)\right| \leq B_{3}|a|^{-3 / 4}|a b|^{-1 / 2}$, if $|a|<q^{-m},|a b|<q^{-m},|b|<1$.

(4) $\left|K_{0}(a, b)\right| \leq B_{3}|a|^{-1 / 2}|a b|^{-3 / 4}$, if $|a|<q^{-m},|a b|<q^{-m},|b|>1$.

Proof.

(1) Notice that $|\gamma(z, \psi)|=1$ for all $z \in F^{*}$ and $\psi$ and that $|(a, b)|=1$ for all $a, b \in F^{*}$. Thus we can choose $B_{1}=|2|^{-1 / 2}$.

(2) The integrand in the definition of $K_{0}$ has absolute value one and the measure of the set $\left\{x \mid b+a x^{2} \equiv 2 P^{m}\right\}$ is bounded by $B_{2}|a|^{-1 / 2}$ for some positive constant $B_{2}$.

(3) can be proved with the use of stationary phase for the integral appearing in $K_{0}$ (see the remark after the statement of Proposition 3.1 in [8]). The stationary phase method gives the bound $B_{3}|a|^{-1 / 4}$ for this integral, where $B_{3}$ is some constant.

(4) comes from (3) using the definition of $K_{0}$.

Jacquet and Ye obtained the following expansions for the $\psi$ orbital integrals. (See $[7,(30)]$.)

Theorem 4.2 ([7, p. 933] and [8, Proposition 3.1]). Fix $f \in C_{c}^{\infty}(G)$ and positive constants $C_{1}, C_{2}, C_{3}$. Let $a \in A$. 
(a) There exist an $\epsilon>0$ and a function $w_{f} \in C_{c}^{\infty}\left(F^{2}\right)$ such that

$$
I_{f, \psi}(a)=\sum_{a=a_{1}(x, y) a_{1}(z)} w_{f}(x, y) K_{1}(z, \psi)
$$

for all a such that $\left|\Delta_{1}(a)\right|<\epsilon,\left|\Delta_{2}(a)\right|=C_{2}$ and $\left|\Delta_{3}(a)\right|=C_{3}$.

Moreover, we can choose $\epsilon$ small enough that $I_{\psi, f}(a)=0$ if a cannot be written in the form $a=a_{1}(x, y) a_{1}(z)$.

(b) There exist an $\epsilon>0$ and a function $w_{f}^{\prime} \in C_{c}^{\infty}\left(F^{2}\right)$ such that

$$
I_{f, \psi}(a)=\sum_{a=a_{2}(x, y) a_{3}(z)} w_{f}^{\prime}(x, y) K_{1}(z, \psi)
$$

for all a such that $\left|\Delta_{1}(a)\right|=C_{1},\left|\Delta_{2}(a)\right|<\epsilon$, and $\left|\Delta_{3}(a)\right|=C_{3}$.

Moreover, we can choose $\epsilon$ to be small enough so that $I_{f, \psi}(a)=0$ if a cannot be written in the form $a=a_{2}(x, y) a_{3}(z)$.

(c) There exist an $\epsilon>0$ and a function $\widetilde{w}_{f} \in C_{c}^{\infty}(F)$ such that if $\left|\Delta_{1}(a)\right|<$ $\epsilon\left|\Delta_{2}(a)\right|<\epsilon$, and $\left|\Delta_{3}(a)\right|=C_{3}$ then

$$
I_{f, \psi}(a)=\sum_{a=z a(x, y)} \widetilde{w}_{f}(z) K_{0}(x, y, \psi)
$$

Moreover, we can choose $\epsilon$ to be small enough so that $I_{f, \psi}(a)=0$ if a cannot be written in the form $a=z a(x, y)$ with $z \in Z$.

Combining Theorem 4.2 and Lemma 4.1 we get:

Corollary 4.3. Fix $f \in C_{c}^{\infty}(G)$. Then $\left|I_{f, \psi}(g) \Delta^{3 / 8}(g)\right|$ is bounded on compact sets in $G$.

Proof. It is enough to show that for every $g \in G$ there exist an open compact neighborhood of $g$ for which the corollary holds. Fix $g_{0} \in G$. If $\Delta_{i}\left(g_{0}\right) \neq 0$, $i=1,2$, we can choose an open compact neighborhood of $g_{0}$ contained in $\bar{N} B$. Since the function $\left|I_{f, \psi}(g) \Delta^{3 / 8}(g)\right|$ is defined and continuous on $\bar{N} B$ the conclusion follows.

Now assume $\Delta_{1}\left(g_{0}\right)=0,\left|\Delta_{2}\left(g_{0}\right)\right|=C_{2} \neq 0$ and $\left|\Delta_{3}\left(g_{0}\right)\right|=C_{3} \neq 0$. We can choose $\epsilon=\epsilon\left(f, C_{2}, C_{3}\right)>0$ as in Theorem 4.2 (a), and a compact neighborhood $Q$ of $g_{0}$ such that $\left|\Delta_{1}(g)\right|<\epsilon,\left|\Delta_{2}(g)\right|=C_{2}$ and $\left|\Delta_{3}(g)\right|=C_{3}$ for all $g \in Q$. Let $g \in Q$ be of the form $g={ }^{t} n_{1} a n_{2}$. Then $a$ satisfies the assumptions in Theorem 4.2 (a) and we have

$$
\left|I_{f, \psi}(g)\right|=\left|I_{f, \psi}(a)\right| \leq C^{\prime} \sum_{a=a_{1}(x, y) a_{1}(z)}\left|K_{1}(z, \psi)\right|
$$


It is easy to see that this sum has at most two summands, and also that $|z|=$ $C_{2}^{-1 / 2}\left|\Delta_{1}(g)\right|=C^{\prime} \Delta^{1 / 2}(g)$, where $C^{\prime}=C_{2}^{-3 / 2} C_{3}$. Hence, by Lemma $4.1(1)$,

$$
\begin{aligned}
\left|I_{f, \psi}(g)\right| \leq B_{1}|z|^{-1 / 2} & \leq B_{2} \Delta^{-1 / 4}(g)=B_{2} \Delta^{-3 / 8}(g) \Delta^{1 / 8}(g) \\
& \leq B_{2} \epsilon^{1 / 4} C_{2}^{1 / 4} C_{3}^{-1 / 4} \Delta^{-3 / 8}(g),
\end{aligned}
$$

where $B_{1}, B_{2}$ are some positive constants independent of $g \in Q$.

If $\left|\Delta_{1}\left(g_{0}\right)\right|=C_{1} \neq 0$ and $\Delta_{2}\left(g_{0}\right)=0$ similar arguments apply.

Now assume $\Delta_{1}\left(g_{0}\right)=0, \Delta_{2}\left(g_{0}\right)=0$ and $\left|\Delta_{3}\left(g_{0}\right)\right|=C_{3}$. We can choose $\epsilon=\epsilon\left(f, C_{3}\right)>0$ as in Theorem 4.2 (c) and a neighborhood $Q$ of $g_{0}$ such that $\left|\Delta_{i}(g)\right|<\epsilon, i=1,2$, and $\left|\Delta_{3}(g)\right|=C_{3}$ for all $g \in Q$. Let $g \in Q$ be of the form $g={ }^{t} n_{1} a n_{2}$. Then $a$ satisfies the assumptions in Theorem 4.2 (c) and we have

$$
\left|I_{f, \psi}(g)\right|=\left|I_{f, \psi}(a)\right| \leq C^{\prime} \sum_{a=z a(x, y)}\left|K_{0}(x, y, \psi)\right| .
$$

Dividing into cases where $|y|=1,|y|<1$ and $|y|>1$ and using parts (2), (3) and (4) of Lemma 4.1, the conclusion follows.

Corollary 4.4. Fix $f \in C_{c}^{\infty}(G)$. Then $\left|I_{f, \omega, \psi}(g) \Delta^{3 / 8}(g)\right|$ is bounded on compact sets in $G$.

Proof. Let $Q_{1}$ be the support of $f$. Since $Q_{1}$ is compact it follows that $|\operatorname{det}(g)|$ is bounded for $g \in Q$. Hence, the support of $I_{f, \psi}$ is also on a set on which the determinant is bounded.

Let $Q_{2}$ be a compact set in $G$. We will show that $\left|I_{f, \omega, \psi}(g) \Delta^{3 / 8}(g)\right|$ is bounded on $Q_{2}$. If $g \in Q_{2}, z \in Z$ and $g z$ is in the support of $I_{\psi, f}$ we have $\operatorname{det}(g z)=\operatorname{det} g \operatorname{det} z$ is in some fixed compact set in $F^{*}$ hence $z$ is in a fixed compact set $P$ in $Z$ independent of $g \in Q_{2}$. Let $C_{1}=\max _{z \in P}(|\omega(z)|)$ and $g \in Q_{2}$. By Corollary 4.3 there exist a constant $C_{2}$ such that $\left|I_{f, \psi}(g z)\right| \leq$ $C_{2} \Delta^{-3 / 8}(g z)$ for all $g \in Q_{2}$ and $z \in P$. Hence, if $g \in Q_{2}$ then

$$
\begin{aligned}
\left|I_{f, \omega, \psi}(g)\right| & =\left|\int_{Z} I_{f, \psi}(g z) \omega^{-1}(z) d z\right|=\left|\int_{P} I_{f, \psi}(g z) \omega^{-1}(z) d z\right| \\
& \leq C_{1} C_{2} \int_{P}\left|\Delta^{-3 / 8}(g z)\right| d z \leq C_{3}\left|\Delta^{-3 / 8}(g)\right| .
\end{aligned}
$$

Here we have used that $\left|\Delta^{-3 / 8}(g z)\right|=\left|\Delta^{-3 / 8}(g)\right|$.

It follows from Corollary 4.4 and from Corollary 3.3 that $I_{f, \omega, \psi}$ is locally integrable, which is the content of Theorem 1.1 stated in the introduction. Consequently, we have proved Corollary 1.2 from the introduction, which states that the Bessel function $j_{\pi, \psi}$, where $\pi$ is an irreducible admissible representation of $G$ with a Whittaker model, is locally integrable. This corollary will be used in the next section to prove the kernel formula promised in the introduction. 


\section{A kernel formula}

Let $N_{2}, A_{2}, K_{2}$ be subgroups of GL(2,F), where $A_{2}$ is the subgroup of diagonal matrices, $K_{2}=\mathrm{GL}(2, O)$ and

$$
N_{2}=\left\{n(x)=\left(\begin{array}{ll}
1 & x \\
& 1
\end{array}\right) \mid x \in F\right\} .
$$

Let

$$
t(r)=\left(\begin{array}{ll}
r & \\
& 1
\end{array}\right) \quad \text { and } \quad w=\left(\begin{array}{ll} 
& 1 \\
1 &
\end{array}\right)
$$

Let

$$
H=\left\{\underline{h}=\left(\begin{array}{ll}
h & \\
& 1
\end{array}\right) \mid h \in \mathrm{GL}(2, F)\right\} .
$$

Let

$$
P=\left\{\left(\begin{array}{ll}
h & v \\
0 & 1
\end{array}\right) \mid h \in \mathrm{GL}(2, F), v \in F^{2}\right\}
$$

and $P_{2}=\left\{n(x) t(r) \mid x \in F, r \in F^{*}\right\}$.

Let $(\pi, V)$ be an irreducible admissible representation of $\mathrm{GL}(3, F)$. Let $L$ be a nonzero $\psi$ Whittaker functional on $V$ (see $[1,1.1]$ ), and let $\mathcal{W}=$ $\mathcal{W}(\pi, \psi)$ be the Whittaker model of $\pi$ consisting of functions of the form $W_{v}, v \in V$, where

$$
W_{v}(g)=L(\pi(g) v)
$$

Let $j_{\pi, \psi}$ be the Bessel function of $\pi . j_{\pi, \psi}$ is defined by equation (1.4) of [1]:

$$
L_{g}(W)=\int_{N}^{\mathcal{N}} W(g n) \psi^{-1}(n) d n=j_{\pi, \psi}(g) W(e),
$$

where $g \in B w_{0} B, W \in \mathcal{W}(\pi, \psi)$ and

$$
\int_{N}^{\mathcal{N}} W(g n) \psi^{-1}(n) d n=\lim _{m \rightarrow \infty} \int_{N_{m}} W(g n) \psi^{-1}(n) d n .
$$

Here $N_{1} \subseteq N_{2} \subseteq \cdots$ is any filtration of $N$ by an ascending chain of open compact subgroups of $N$. It follows from [1, Corollary 1.3] that the limit above stabilizes for large $m$.

Let $\mathcal{W}^{0}=\mathcal{W}^{0}(\pi, \psi)$ be the subspace of $\mathcal{W}$ defined in [1, Definition 4.1]. It follows from $\left[\mathbf{1}\right.$, Theorem 4.6] that if $W \in \mathcal{W}^{0}$ and $g \in B w_{0} B$ the function

$$
n \mapsto W(g n)
$$

is compactly supported in $N$, and hence we can write

$$
L_{g}(W)=\int_{N} W(g n) \psi^{-1}(n) d n=j_{\pi, \psi}(g) W(e),
$$

where in this case we use the standard integration. 
Lemma 5.1. Let $W \in \mathcal{W}^{0}$. Then the function

$$
h \mapsto W(\underline{h})
$$

from $\mathrm{GL}(2, F)$ to $\mathbf{C}$ is compactly supported $\bmod N_{2}$.

Proof. Each $\underline{h} \in H$ is in a Bruhat cell $B w B$ with $S^{0}(w)=\left\{\alpha_{1,2}, \alpha_{2,3}\right\}$ (see $[\mathbf{1}, 4.2])$. We write the Iwasawa decomposition of $\underline{h}$

$$
\underline{h}=\underline{n} \underline{a} \underline{k},
$$

where $h=n a k, h \in \mathrm{GL}(2, F), n \in N_{2}, a \in A_{2}, k \in K_{2}$.

Let $a=\operatorname{diag}\left(a_{1}, a_{2}\right) \in A_{2}$ and $\underline{a}=\operatorname{diag}\left(a_{1}, a_{2}, 1\right)$. By the definition of $\mathcal{W}^{0}$ in $\left[\mathbf{1}\right.$, Definition 4.1] we have that if $W \in \mathcal{W}^{0}$ and $W(\underline{h}) \neq 0$ then $\alpha_{1,2}(\underline{a})=a_{1} / a_{2}$ is in a compact set in $F^{*}$ and $\alpha_{2,3}(\underline{a})=a_{2}$ is in a compact set in $F^{*}$, where both compact sets are independent of $n$ and $k$ in the Iwasawa decomposition of $h$. Hence $a_{1}$ and $a_{2}$ are in a compact set in $F^{*}$ and it follows that the support of $W$ on $H$ is compact $\bmod N_{2}$.

The next proposition follows from [1, Theorem 4.6].

Proposition 5.2. Let $W \in \mathcal{W}^{0}$ and $h \in \mathrm{GL}(2, F)$ then the function

$$
v \mapsto W\left(\begin{array}{cc} 
& 1 \\
h &
\end{array}\right)\left(\begin{array}{ll}
I & v \\
& 1
\end{array}\right)
$$

from $F^{2}$ to $\mathbf{C}$ is compactly supported in $F^{2}$.

Lemma 5.3. Let $W \in \mathcal{W}$ be such that

$$
h \mapsto W(\underline{h})
$$

is compactly supported mod $N_{2}$ Then

$$
\int_{N_{2} \backslash \mathrm{GL}(2, F)} j_{\pi, \psi}\left(\begin{array}{ll} 
& 1 \\
g h^{-1} &
\end{array}\right) W\left(\begin{array}{ll}
h & \\
& 1
\end{array}\right) d h
$$

converges absolutely for all $g \in \mathrm{GL}(2, F)$.

Proof. Since changing variables $h \mapsto g h^{-1}$ amounts to changing $W$ to a different $W$ satisfying the same hypothesis we can assume that $g=e$.

We let $Y_{1}$ be the open dense set of $G$ given by elements of the form

$$
\left(\begin{array}{cc}
I & u_{1} \\
& 1
\end{array}\right)\left(\begin{array}{cc} 
& r \\
h &
\end{array}\right)\left(\begin{array}{cc}
I & u_{2} \\
& 1
\end{array}\right)
$$

where $u_{1}, u_{2} \in F^{2}, h \in \mathrm{GL}(2, F)$ and $r \in F^{*}$.

Let $d x=d u_{1}|\operatorname{det} h| d h|r|^{-2} d^{*} r d u_{2}$ be a Haar measure on GL(3, $\left.F\right)$ restricted to $Y_{1}$. Since $j_{\pi, \psi}$ is locally integrable on $Y$ it follows that the function $g \mapsto j_{\pi, \psi}\left(g^{-1}\right)$ is locally integrable on $G$. Hence, if $f \in C_{c}^{\infty}\left(Y_{1}\right)$ then

$$
\int_{\mathrm{GL}(2, F)} j_{\pi, \psi}\left(h_{h^{-1}}{ }^{1}\right) f\left(\begin{array}{ll}
{ }_{h} & 1
\end{array}\right)|\operatorname{det} h| d h
$$


is absolutely convergent. Since $f \in C_{c}^{\infty}\left(Y_{1}\right)$, it means that the function $|\operatorname{det} h|$ appearing in the above integral is bounded on the support of $f$. Hence we can drop $|\operatorname{det} h|$ in the integral and still have absolute convergence. Integrating by steps we have

$$
\begin{aligned}
& \int_{\mathrm{GL}(2, F)} j_{\pi, \psi}\left(\begin{array}{ll}
h^{-1} & 1
\end{array}\right) f\left(\begin{array}{ll}
h^{1} & 1
\end{array}\right) d h \\
& =\int_{N_{2} \backslash \mathrm{GL}(2, F)} j_{\pi, \psi}\left(\begin{array}{ll}
h^{-1} & 1
\end{array}\right)\left(\int_{N_{2}} f\left({ }_{n h}{ }^{1}\right) \psi^{-1}(n) d n\right) d h .
\end{aligned}
$$

Here, the measure $d h$ on the right-hand side is a Haar measure on $\operatorname{GL}(2, F)$ and the measure $d h$ on the left side is a $\operatorname{GL}(2, F)$ invariant measure on $N_{2} \backslash \mathrm{GL}(2, F)$. We view the inner integral as a function on $\operatorname{GL}(2, F)$. It is well-known (see [1, Lemma 7.1]) that any smooth function on $\mathrm{GL}(2, F)$ that is compactly supported mod $N_{2}$ and is $\psi$ equivariant under left translations by $N_{2}$ can be obtained this way, in particular the function $h \mapsto W(\underline{h})$ is such a function.

Our main theorem of this section is the following:

Theorem 5.4. Let $W \in \mathcal{W}^{0}$ and $y \in \operatorname{GL}(2, F)$. Assume further that $y \in$ $B_{2} w B_{2}$. Then

$$
W\left(\begin{array}{ll} 
& 1 \\
y &
\end{array}\right)=\int_{N_{2} \backslash \mathrm{GL}(2, F)} j_{\pi, \psi}\left(\begin{array}{ll}
y h^{-1} & 1
\end{array}\right) W\left(\begin{array}{ll}
h & \\
& 1
\end{array}\right) d h .
$$

Proof. By Lemma 5.1 and Lemma 5.3 this integral converges. For each $W \in \mathcal{W}^{0}$ and $y \in \mathrm{GL}(2, F)$ we define a function $M_{W}: \mathrm{GL}(2, F) \rightarrow \mathbf{C}$ by

$$
M_{W}(y)=\int_{F^{2}} W\left(\begin{array}{ll} 
& 1 \\
y &
\end{array}\right)\left(\begin{array}{ll}
I & u \\
& 1
\end{array}\right) \psi(-u) d u
$$

where $u={ }^{t}\left(u_{1}, u_{2}\right)$ is a column vector and $\psi(u)=\psi_{F}\left(u_{2}\right)$. By Proposition 5.2, this integral converges. For $h \in \mathrm{GL}(2, F)$ we define $\psi^{h}(u)=\psi(h u)$. We have

$$
\begin{aligned}
& \int_{F^{2}} W\left(\begin{array}{ll} 
& 1 \\
y &
\end{array}\right)\left(\begin{array}{ll}
I & u \\
& 1
\end{array}\right) \psi^{h}(-u) d u \\
& =\int_{F^{2}} W\left(\begin{array}{l}
1 \\
y
\end{array}\right)\left(\begin{array}{cc}
I & h^{-1} u \\
& 1
\end{array}\right)|\operatorname{det} h|^{-1} \psi(-u) d u \\
& =\int_{F^{2}} W\left(\begin{array}{ll} 
& \\
y h^{-1} &
\end{array}\right)\left(\begin{array}{ll}
I & u \\
& 1
\end{array}\right)\left(\begin{array}{ll}
h & \\
& 1
\end{array}\right)|\operatorname{det} h|^{-1} \psi(-u) d u \\
& =|\operatorname{det} h|^{-1} M_{\pi(h) W}\left(y h^{-1}\right) \text {. }
\end{aligned}
$$


Let $F^{2}$ be the space of column vectors and let $f \in C_{c}^{\infty}\left(F^{2}\right)$. Let $\psi$ be a character on $F^{2}$ as above. Define $\hat{f}: \operatorname{GL}(2, F) \rightarrow \mathbf{C}$ by

$$
\hat{f}(h)=\int_{F^{2}} f(v) \psi^{h}(-v) d v .
$$

It is easy to see that $f(p h)=f(h)$ for every $p \in P_{2}, h \in \mathrm{GL}(2, F)$. Using the identification of $P_{2} \backslash \mathrm{GL}(2, F)$ with $F^{2}-\{0\}$ it is easy to see that $\hat{f}$ is exactly the Fourier transform of $f$. Since the $\mid$ det $\mid$ equivariant measure $d h$ on $P_{2} \backslash \mathrm{GL}(2, F)$ is identified with the measure $d v$ on $F^{2}-\{0\}$ we can use the standard Fourier inversion formula to conclude that

$$
f(u)=\int_{P_{2} \backslash \mathrm{GL}(2, F)} \hat{f}(h) \psi^{h}(u) d h .
$$

In particular taking $u=0$ we get

$$
f(0)=\int_{P_{2} \backslash \mathrm{GL}(2, F)} \hat{f}(h) d h .
$$

We now pick $f$ to be the function

$$
u \mapsto W\left(\begin{array}{ll} 
& 1 \\
y &
\end{array}\right)\left(\begin{array}{ll}
I & u \\
& 1
\end{array}\right)
$$

and get

$$
W\left(\begin{array}{ll}
y & 1 \\
y &
\end{array}\right)=\int_{P_{2} \backslash \mathrm{GL}(2, F)} M_{\pi(h) W}\left(y h^{-1}\right)|\operatorname{det} h| d h .
$$

It follows from [1, Theorem 4.6] that the function

$$
x \mapsto M_{W}(y n(x))
$$

from $F$ to $\mathbf{C}$ is compactly supported for every fixed $y \in B_{2} w B_{2}$, where $w$ is the long Weyl element of $\mathrm{GL}(2, F)$ that was defined in the beginning of this section. It is easy to see that

$$
\int M_{W}(y n(x)) \psi^{-1}(x) d x=\int_{N} W\left(\left(\begin{array}{ll}
y & 1
\end{array}\right) n\right) \psi^{-1}(n) d n .
$$

Hence by (5.3) we have

$$
\int M_{W}(y n(x)) \psi^{-1}(x) d x=j_{\pi, \psi}\left(\begin{array}{l}
1 \\
y
\end{array}\right) W(I) .
$$

An argument similar to the one above shows that

$$
\int M_{W}(y n(x)) \psi^{-1}(a x) d x=j_{\pi, \psi}\left(\begin{array}{ll} 
& 1 \\
y &
\end{array}\right)\left(\begin{array}{ll}
t(a) & \\
& 1
\end{array}\right) W\left(\underline{t\left(a^{-1}\right)}|a|^{-1} .\right.
$$

Applying Fourier inversion to the function

$$
f(x)=M_{W}(y n(x))
$$


we get

$$
M_{W}(y)=\int j_{\pi}\left(\begin{array}{ll} 
& 1 \\
y &
\end{array}\right)\left(\begin{array}{ll}
\frac{t(a)}{} & \\
& 1
\end{array}\right) W\left(\underline{t\left(a^{-1}\right)}\right) d^{*} a .
$$

Combining (5.5) with (5.6) we get

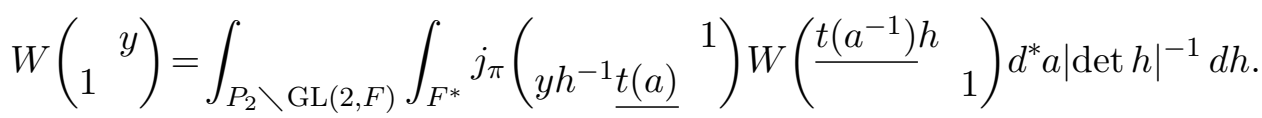

If $f$ is an absolutely integrable function on $N_{2} \backslash \mathrm{GL}(2, F)$ then

$$
\int_{N_{2} \backslash \mathrm{GL}(2, F)} f(m) d m=\int_{P_{2} \backslash \mathrm{GL}(2, F)}\left(\int_{F^{*}} f(t(a) h) d^{*} a\right)|\operatorname{det} h|^{-1} d h,
$$

where $d m$ is a $\operatorname{GL}(2, F)$ invariant measure on $N_{2} \backslash \mathrm{GL}(2, F)$ and $d h$ is a $\mid$ det $\mid$ equivariant measure on $P_{2} \backslash \mathrm{GL}(2, F)$ with an appropriate normalization. Hence the integral above matches the integral in the statement.

Corollary 5.5. Let $g \in B w_{0} B$ and $W \in \mathcal{W}^{0}$. Then

$$
W(g)=\int_{N_{2} \backslash \mathrm{GL}(2, F)} j_{\pi}\left(g \underline{h}^{-1}\right) W(\underline{h}) d h .
$$

Proof. By Theorem 5.4, this statement is true for all $g \in B w_{0} B$ of the form

$$
g=\left(\begin{array}{cc} 
& 1 \\
g_{0} &
\end{array}\right)
$$

with $g_{0} \in B_{2} w B_{2}$. Using the equivariance of $W$ and $j_{\pi, \psi}$ under $N$ and $Z$ we get the statement for all $g \in B w_{0} N_{\alpha_{1,2}}$, where

$$
N_{\alpha_{1,2}}=\left\{\left(\begin{array}{ccc}
1 & x & \\
& 1 & \\
& & 1
\end{array}\right) \mid x \in F\right\} .
$$

Let

$$
n=\left(\begin{array}{ll}
I & u \\
& 1
\end{array}\right),
$$

with $u \in F^{2}$. Then $\pi(n) W \in \mathcal{W}^{1}$. Let $g \in B w_{0} N_{\alpha_{1,2}}$. Then

$$
(\pi(n) W)(g)=\int_{N_{2} \backslash \mathrm{GL}(2, F)} j_{\pi}\left(g \underline{h}^{-1}\right)(\pi(n)) W(\underline{h}) d h .
$$

Hence

$$
\begin{aligned}
W(g n) & =\int_{N_{2} \backslash \mathrm{GL}(2, F)} j_{\pi}\left(g \underline{h}^{-1}\right) W(\underline{h} n) d h \\
& =\int_{N_{2} \backslash \mathrm{GL}(2, F)} j_{\pi}\left(g \underline{h}^{-1}\right) \psi\left(\underline{h} n \underline{h}^{-1}\right) W(\underline{h}) d h \\
& =\int_{N_{2} \backslash \mathrm{GL}(2, F)} j_{\pi}\left(g n \underline{h}^{-1}\right) W(\underline{h}) d h .
\end{aligned}
$$


Corollary 5.6. Let $\hat{\pi}$ be a the contragredient representation to $\pi$ and let $\hat{W} \in \mathcal{W}^{0}\left(\hat{\pi}, \psi^{-1}\right)$. Let $g \in B w_{0} B$. Then

$$
\hat{W}\left(g^{-1}\right)=\int_{N_{2} \backslash \mathrm{GL}(2, F)} j_{\pi, \psi}(\underline{h} g) \hat{W}(\underline{h}) d h .
$$

Proof. By [1, Corollary 6.12], $j_{\hat{\pi}, \psi^{-1}}(g)=j_{\pi, \psi}\left(g^{-1}\right)$. We get our result by applying this to Corollary 5.5.

\section{Bessel Distributions are given by Bessel functions}

In this section we prove the main result of this work. We shall show that for $G=\mathrm{GL}(3, F)$ the Bessel distributions are given by Bessel functions. In [2] we showed using a different method that Bessel distributions for quasisplit reductive groups over local fields are given by Bessel functions on the open cell. The following result provides more evidence toward the conjecture that Bessel distributions are given by Bessel functions on the whole group.

Let $(\pi, V)$ be an irreducible admissible representation of $G$ with a $\psi$ Whittaker functional $L$ and let $(\hat{\pi}, \hat{V})$ be the representation contragredient to $\pi$. We think of $\hat{V}$ as the space of smooth linear functionals on $V$. Let $\hat{L}$ be a $\psi^{-1}$ Whittaker functional on $\hat{V}$. It follows from [3] that we can (and will) normalize $\hat{L}$ so that if $v \in V$ and $\hat{v} \in \hat{V}$ are such that either $g \mapsto W_{v}(\underline{h})$ or $g \mapsto \hat{W}_{\hat{v}}(\underline{h})$ have compact support in $\operatorname{GL}(2, F) \bmod N_{2}$, then

$$
\hat{v}(v)=\langle\hat{v}, v\rangle=\int_{N_{2} \backslash \mathrm{GL}(2, F)} W_{v}(\underline{h}) \hat{W}_{\hat{v}}(\underline{h}) d h .
$$

Here $W_{v} \in \mathcal{W}(\pi, \psi)$ and $\hat{W}_{\hat{v}} \in \mathcal{W}\left(\hat{\pi}, \psi^{-1}\right)$ are defined as in (5.1). The assumption on the support is made to ensure that the integral converges. It is well-known that $\hat{\hat{\pi}} \cong \pi$. We identify $V$ and $\hat{\hat{V}}$. For a linear functional (smooth or not) $T: \hat{V} \mapsto \mathbb{C}$ and $f \in C_{c}^{\infty}(G)$ we define the linear functional $\rho(f) T: \hat{V} \mapsto \mathbb{C}$ by

$$
(\rho(f) T)(\hat{v})=\int_{G} f(g) T\left(\hat{\pi}\left(g^{-1}\right) v\right) d g, \quad \hat{v} \in \hat{V} .
$$

It is clear that $\rho(f) T$ is a smooth linear functional hence we can identify $\rho(f) T$ with a vector $v_{f, T} \in V$. We now define the Bessel distribution (first defined by Gelfand and Kazhdan in [5]), $J_{\pi}: C_{c}^{\infty}(G) \mapsto \mathbb{C}$ to be

$$
J_{\pi}(f)=\langle L, \rho(f) \hat{L}\rangle=L\left(v_{f, \hat{L}}\right) .
$$

Lemma 6.1. Let $\hat{v} \in \hat{V}$ be such that $h \mapsto \hat{W}_{\hat{v}}(\underline{h})$ has compact support mod $N_{2}$ in $\mathrm{GL}(2, F)$. Then

$$
\int_{N_{2} \backslash \mathrm{GL}(2, F)} J_{\pi}\left(\rho_{l}(\underline{h}) f\right) \hat{W}_{\hat{v}}(\underline{h}) d h=\int_{G} f(g) \hat{W}_{\hat{v}}\left(g^{-1}\right) d g .
$$


Proof. By definition, $J_{\pi}\left(\rho_{l}(\underline{h}) f\right)=W_{v_{f, \hat{L}}}(\underline{h})$. Hence, by (6.1), the left-hand side of (6.4) is the same as $\left\langle\hat{v}, v_{f, L}\right\rangle$, which equals $\langle\hat{v}, \rho(f) \hat{L}\rangle$. By (6.2) this is exactly the right-hand side of (6.4).

Let $j_{\pi, \psi}$ be the Bessel function of $\pi$. (See (5.2)). The main theorem of this paper is the following:

\section{Theorem 6.2.}

$$
J_{\pi}(f)=\int_{G} f(g) j_{\pi, \psi}(g) d g, \quad f \in C_{c}^{\infty}(G) .
$$

Proof. We define the distribution $J_{\pi, 1}$ on $C_{c}^{\infty}(G)$ to be

$$
J_{\pi, 1}(f)=\int_{G} f(g) j_{\pi, \psi}(g) d g, \quad f \in C_{c}^{\infty}(G) .
$$

By Corollary $1.3, j_{\pi, \psi}$ is locally integrable, hence $J_{\pi, 1}$ is well-defined. We shall prove that $J_{\pi, 1}=J_{\pi}$.

Let $f \in C_{c}^{\infty}(G)$. There exist an integer $m$ such that $\rho_{l}(\underline{h}) f=f$ for all $h \in K_{2}^{m}$. Here $\rho_{l}$ is left translation and $K_{2}^{m}=I_{2}+M_{2}\left(P^{m}\right)$. Let

$$
\bar{B}_{2, n}=\left\{\left(\begin{array}{cc}
x_{1} & 0 \\
x_{2} & x_{3}
\end{array}\right) \mid x_{1}, x_{3} \in 1+P^{n}, x_{2} \in P^{3 n}\right\} .
$$

It follows from [1, Theorem 5.7, Lemma 5.1, Lemma 5.4] that there exist $\hat{W} \in \mathcal{W}^{0}(\hat{\pi}, \psi)$ and $n>m$ such that the function

$$
h \mapsto \hat{W}(\underline{h})
$$

is supported on $N_{2} \bar{B}_{2, n}$ and such that

$$
\hat{W}(n \bar{b})=\frac{1}{\operatorname{vol}\left(\bar{B}_{2, n}\right)} \psi^{-1}(n)
$$

for every $n \in N_{2}$ and $\bar{b} \in \bar{B}_{2, n}$. We have

$$
\int_{N_{2} \backslash \operatorname{GL}(2, F)} J_{\pi, 1}\left(\rho_{l}(\underline{h}) f\right) \hat{W}(\underline{h}) d h=\int_{\bar{B}_{2, n}} J_{\pi, 1}\left(\rho_{l}(\underline{\bar{b}}) f\right) \hat{W}(\underline{\bar{b}}) d_{r} \bar{b}=J_{\pi, 1}(f),
$$

where $d_{r} \bar{b}$ is a right invariant Haar measure on $\bar{B}_{2}$. Thus we have

$$
\begin{aligned}
J_{\pi, 1}(f) & =\int_{N_{2} \backslash \mathrm{GL}(2, F)} J_{\pi, 1}\left(\rho_{l}(\underline{h}) f\right) \hat{W}(\underline{h}) d h \\
& =\int_{N_{2} \backslash \mathrm{GL}(2, F)} \hat{W}(\underline{h})\left(\int_{G} f\left(\underline{h}^{-1} g\right) j_{\pi, \psi}(g) d g\right) d h \\
& =\int_{N_{2} \backslash \mathrm{GL}(2, F)} \hat{W}(\underline{h})\left(\int_{G} f(g) j_{\pi, \psi}(\underline{h} g) d g\right) d h \\
& =\int_{G} f(g)\left(\int_{N_{2} \backslash \mathrm{GL}(2, F)} j_{\pi, \psi}(\underline{h} g) \hat{W}(\underline{h}) d h\right) d g=\int_{G} f(g) \hat{W}\left(g^{-1}\right) d g .
\end{aligned}
$$


We notice that $\int_{G} f\left(\underline{h}^{-1} g\right) j_{\pi, \psi}(g) d g$ is absolutely convergent and that the absolute integral gives a locally constant function in the variable $h$. Since $\hat{W}(\underline{h})$ is compactly supported mod $N_{2}$ we get absolute convergence for the iterated integral hence we can use Fubini's theorem to change the order. The $G$ integration is in fact taking place on the open and dense set $B w_{0} B$, hence we can use Corollary 5.6 to obtain the last equality. By (6.4) this last integral equals

$$
\int_{N_{2} \backslash \mathrm{GL}(2, F)} J_{\pi}\left(\rho_{l}(\underline{h} f) \hat{W}(\underline{h}) d h\right.
$$

and using the assumptions on $\hat{W}$ as above we get that this integral equals $J_{\pi}(f)$.

\section{References}

[1] E.M. Baruch, Bessel functions for GL(3) over a p-adic field, Pacific J. of Math., 211(1) (2003), 1-33, MR 2016587.

[2] E.M. Baruch, On Bessel distributions for quasi-split groups, Trans. Amer. Math. Soc., 353(7) (2001), 2601-2614, MR 1828462 (2002b:22029), Zbl 0976.22015.

[3] J.N. Bernstein, P-invariant distributions on $\mathrm{GL}(N)$ and the classification of unitary representations of $\mathrm{GL}(N)$ (non-Archimedean case), in 'Lie group representations, II' (College Park, Md., 1982/1983), Lecture Notes in Math., 1041, 50-102, Springer, Berlin, 1984, MR 0748505 (86b:22028), Zbl 0541.22009.

[4] R. Dạbrowski and M. Reeder, Kloosterman sets in reductive groups, J. Number Theory, 73(2) (1998), 228-255, MR 1658031 (2000b:11097), Zbl 0919.11055.

[5] I.M. Gelfand and D.A. Kajdan, Representations of the group $\operatorname{GL}(n, K)$ where $K$ is a local field, in 'Lie groups and their representations' (Proc. Summer School, Bolyai János Math. Soc., Budapest, 1971), 95-118, Halsted, New York, 1975, MR 0404534 (53 \#8334), Zbl 0348.22011.

[6] Harish-Chandra, Harmonic analysis on reductive p-adic groups, Lecture Notes in Math., 162, Springer-Verlag, Berlin, 1970, Notes by G. van Dijk, MR 0414797 (54 \#2889), Zbl 0202.41101.

[7] H. Jacquet and Y. Ye, Distinguished representations and quadratic base change for GL(3), Trans. Amer. Math. Soc., 348(3) (1996), 913-939, MR 1340178 (96h:11041), Zbl 0861.11033.

[8] H. Jacquet and Y. Ye, Germs of Kloosterman integrals for GL(3), Trans. Amer. Math. Soc., 351(3) (1999), 1227-1255, MR 1443878 (99j:11053), Zbl 0919.11038.

Received March 11, 2003. The author was partially supported by NSF grant DMS0070762 .

Department of Mathematics

University of CaLifornia Santa CRUZ

SANTA CRuz CA 95064

E-mail address: baruch@math.ucsc.edu 